\title{
Influence of Surface Clutter on THz Spectroscopy of Skin
}

\author{
G. M. Png*, B. W.-H. Ng*, J. W. Choi ${ }^{\dagger}$, X.-C. Zhang ${ }^{\dagger}$, D. Abbott* \\ ${ }^{*}$ School of Electrical \& Electronic Engineering, The University of Adelaide, SA 5005, Australia \\ $\dagger$ Department of Physics, Applied Physics and Astronomy, Rensselaer Polytechnic Institute, Troy, NY, USA \\ Email: gpng@eleceng.adelaide.edu.au
}

\begin{abstract}
The presence of hair follicles in human skin appear not to disturb Terahertz (THz) transmission, but existing measurements made on human head hair show conflicting results on THz scattering. It is not known if large numbers of hair strands protruding from skin would affect $\mathrm{THz}$ transmission. This scenario is distinct from measuring clumps of head hair because hair strands on skin (particularly on animals) are not bound together, are oriented randomly, are mostly thinner than head hair, and have slightly varying lengths. This study presents measured $\mathrm{THz}$ data on excised rat skin before and after shortening/removal of hair. Results show that the $\mathrm{THz}$ signals before and after hair shortening/removal are similar. A mathematical model is also presented to elucidate the effects of rough surfaces on $\mathbf{T H z}$ reflection mode spectroscopy.
\end{abstract}

\section{INTRODUCTION}

Many samples that are analyzed using Terahertz (THz) Time Domain Spectroscopy (THz-TDS) can be assumed to have ideal surfaces where very little incident $\mathrm{THz}$ is lost through unwanted reflection, such as dispersed and specular reflection. If however a sample has a "rough" surface, then how much do surface irregularities influence unwanted reflections? We present a study that first looks at the effect of hair on $\mathrm{THz}$ transmission through a sample of lyophilized rat skin, where the result shows negligible influence. The study then introduces two models for modeling irregular surfaces, and uses one of these models to quantify the amount of unwanted reflection from very smooth and very rough skin. The results from the latter part of this study are consistent with the empirical observation.

\section{EXPERIMENT AND RESULTS}

Lyophilized ventral skin from a white lab rat was analyzed using transmission mode THz-TDS based on a $100 \mathrm{fs}, 800$ nm Ti:Sapphire laser (Coherent ${ }^{\mathrm{TM}}$ Mira 900). Details of a THzTDS system can be found in [1]. Lyophilized skin was used instead of fresh skin to eliminate the effects of dehydration on the measured $\mathrm{THz}$ signal. The sample was $1 \mathrm{~mm}$ thick with hair strands on one side. Lengths of the hair strands were in the order of the longer $\mathrm{THz}$ wavelengths $(3-0.3 \mathrm{~mm})$. The sample was held at the edges so that $\mathrm{THz}$ radiation could be directed at near normal incidence on the hair side. Several measurements were made and averaged before the sample was shaved as cleanly as possible and the measurements repeated.

Fig. 1 shows the extracted optical properties from 0.3 to 1 $\mathrm{THz}$ for the two scenarios highlighted above. The plots of the extinction coefficients deviate by no more than 0.01 at each frequency, thus are quite similar. The plots of the refractive indices may initially appear very different but considering the small range of values in the ordinate ( $y$-axis), their refractive indices are not dissimilar. The removal of hair appears to have not altered the sample's transmissivity, although it is noted here that hair follicles embedded in the skin were not removed thus the surface was not entirely smooth.

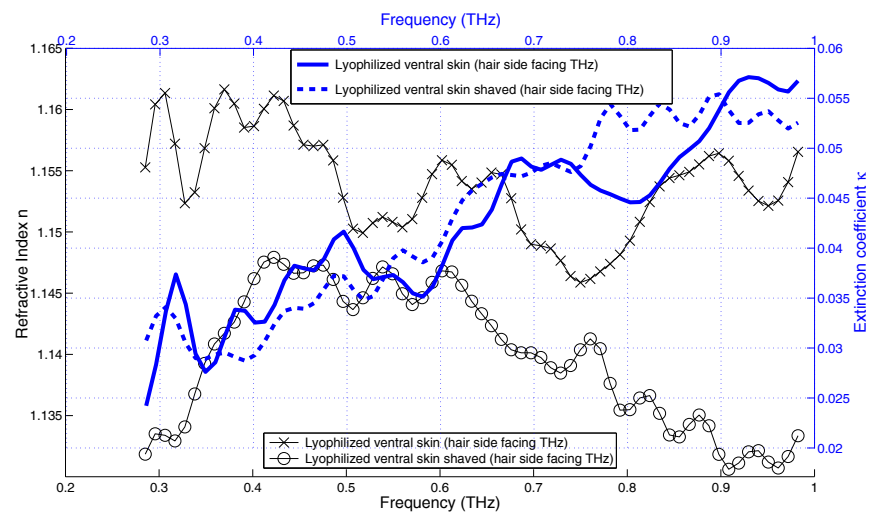

Fig. 1. Optical properties of rat ventral skin, with and without removal of hair. The solid and dashed lines represent the extinction coefficients, while the lines with circle and cross markers represent the refractive indices.

\section{MODElS FOR IRREGULAR SURFACES}

In order to understand the empirical observations in Section II, models of irregular surfaces were explored. The theory of the interaction of electromagnetic radiation with rough surfaces has been studied extensively in areas such as radar and computer graphics [2]-[4]. Studies in the $\mathrm{THz}$ regime have recently emerged [5], [6].

Surface roughness is often modeled as an array of microfacets on an otherwise flat surface. The slopes and spacings of these facets are defined as a distribution function (probability density function). One simple model is the Gaussian model where roughness is altered via changes to the standard deviation of a Gaussian distribution function. In accordance with literature, this standard deviation is called the distribution factor $m$-small $m$ implies smoothness, while large $m$ indicates roughness. Each microfacet has a unit surface normal N; the incoming radiation with respect to $\mathbf{N}$ is unit vector $\mathbf{L}$; the viewing direction with respect to $\mathbf{N}$ is unit vector $\mathbf{V}$; the vector 
that bisects $\mathbf{L}$ and $\mathbf{V}$ is $\mathbf{H}$. The angle of incidence between $\mathbf{N}$ and $\mathbf{H}$ is $\alpha$.

A similar but more thorough model called the Beckmann distribution function [2] is used in this study. Its simplified form is given in (1). To account for some facets that may be blocked from the incident radiation by other facets, a geometric attenuation factor $G$ as given in (2) is incorporated.

$$
\begin{aligned}
& D=\frac{1}{m^{2} \cos ^{4} \alpha} e^{-\left(\frac{\tan \alpha}{m}\right)^{2}} . \\
& G=\min \left(1, \frac{2(\mathbf{N} \cdot \mathbf{H})(\mathbf{N} \cdot \mathbf{V})}{\mathbf{V} \cdot \mathbf{H}}, \frac{2(\mathbf{N} \cdot \mathbf{H})(\mathbf{N} \cdot \mathbf{L})}{\mathbf{V} . \mathbf{H}}\right) .
\end{aligned}
$$

The scene size used in this simulation is $128 \times 128$ pixels. The amount of specular versus diffuse reflection can be altered using the factors $s$ and $d$ respectively, where $s+d=1$. The sample simulated in this study is ideal fresh skin (no moisture) with optical properties consistent with those reported in [7]. The Fresnel reflection coefficient $F(\theta, \lambda)$ of the sample can therefore be calculated, where $\lambda$ is the wavelength and $\theta$ is the angle of incidence between $\mathbf{L}$ and $\mathbf{N}$. The skin sample is assumed to be slightly convex in the direction of the incoming $\mathrm{THz}$ radiation in order to mimic actual skin samples that are seldom flat. The solid angle $\Omega$ of the incident $\mathrm{THz}$ radiation $I_{i}(\lambda)$ is calculated over $2 \times 2$ pixels. The reflected $\mathrm{THz}$ signal $I_{r}(\lambda)$ in the frequency domain is therefore

$$
I_{r}(\lambda)=I_{i}(\lambda)(\mathbf{N} . \mathbf{L}) \Omega\left(\frac{s F(\theta, \lambda) D G}{\pi(\mathbf{N} . \mathbf{V})(\mathbf{N} . \mathbf{L})}+\frac{d F\left(\theta=0^{\circ}, \lambda\right)}{\pi}\right) .
$$

Fig. 2 and Fig. 3 show the magnitude of $I_{r}$ at $1.225 \mathrm{THz}$ for very smooth and very rough skin surfaces respectively. The signal reflected from a rough surface is one order of magnitude less than for a smooth surface, implying less signal return. This is consistent with the notion of more loss from repeated bounces off the facets and is in agreement with [6] where rough surfaces attenuate the detected $\mathrm{THz}$ signal in reflection mode $\mathrm{THz}$ spectroscopy. Since the empirical results in Section II were similar, the conclusion is that skin without hair is still almost as rough as skin with short hair, thus removal of short hair is not critical when measuring skin.

The reflected signal is assumed to be reflected by $180^{\circ}$ for simplicity and the resulting signal in the time domain is as shown in Fig. 4.

\section{CONCLUSION}

This study has shown via empirical data and a mathematical model that removal of short hair is not critical to $\mathrm{THz}$ measurements of rat skin. Future work will look into phase changes due to surface roughness and more advanced models to incorporate the orientation of long hair.

\section{REFERENCES}

[1] P. Siegel, "Terahertz technology," IEEE Transactions on Microwave Theory and Techniques, vol. 50, no. 3, pp. 910-928, 2002.

[2] P. Beckmann and A. Spizzichino, The Scattering of Electromagnetic Waves from Rough Surfaces, ser. Electromagnetic Waves. London: Pergamon Press Ltd., 1963, vol. 4.

[3] K. Torrance and E. Sparrow, "Theory for off-specular reflection from roughened surfaces," Journal of the Optical Society of America, vol. 57, no. 9, pp. 1105-1114, 1967.

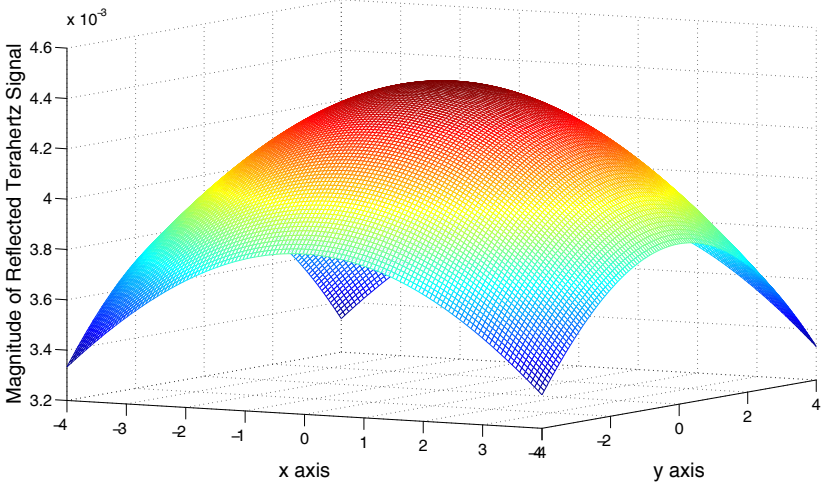

Fig. 2. Magnitude of the reflected $\mathrm{THz}$ signal at $1.225 \mathrm{THz}$ for a smooth surface $(m=0.1), \mathrm{s}=0.1, \mathrm{~d}=0.9$. The maximum magnitude at $4.6 \times 10^{-3}$ (shown in dark red) indicates maximum reflectivity.

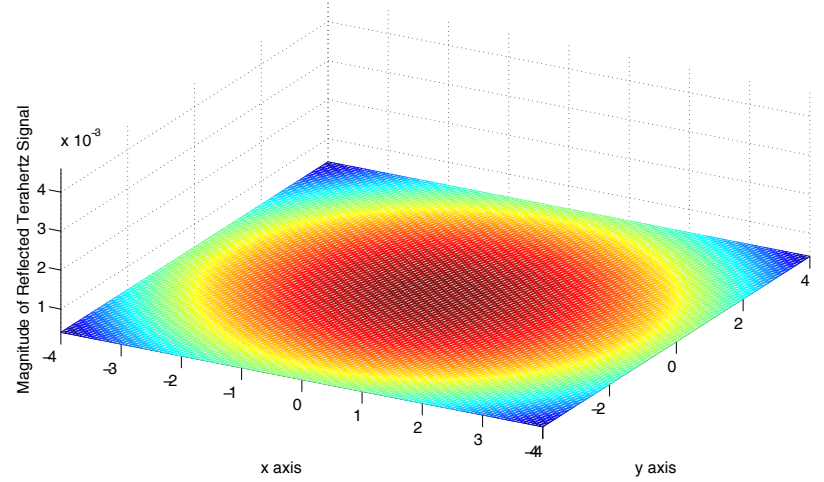

Fig. 3. Magnitude of the reflected $\mathrm{THz}$ signal at $1.225 \mathrm{THz}$ for a rough surface $(m=1), \mathrm{s}=0.1, \mathrm{~d}=0.9$. The maximum magnitude is $4.06 \times 10^{-4}$.
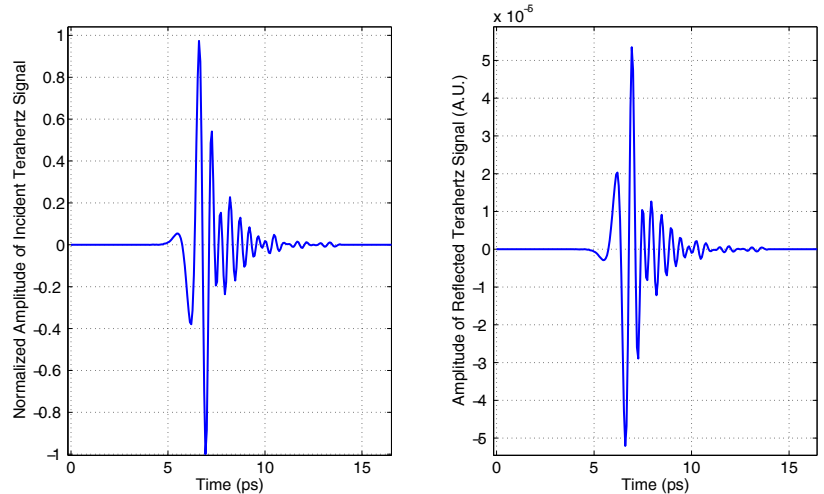

Fig. 4. Measured incident $\mathrm{THz}$ signal and simulated reflected $\mathrm{THz}$ signal using the Beckmann distribution function with $m=1$.

[4] J. Ogilvy, Theory of Wave Scattering from Random Rough Surfaces. Bristol, England: IOP Publishing Ltd., 1991.

[5] G. Png, B.-H. Ng, S. Mickan, D. Abbott, J.-W. Choi, S. Sengupta, and I. Wilke, "Creams and oils: Possible THz coupling media for rough surfaces?" in IRMMW-THz, Shanghai, China, 2006, p. 445.

[6] Y. Dikmelik, J. Spicer, M. Fitch, and R. Osiander, "Effects of surface roughness on reflection spectra obtained by terahertz time-domain spectroscopy," Optics Letters, vol. 31, no. 24, pp. 3653-3655, 2006.

[7] A. Fitzgerald, E. Berry, N. Zinov'ev, S. Homer-Vanniasinkam, R. Miles, J. Chamberlain, and M. Smith, "Catalogue of human tissue optical properties at terahertz frequencies," Journal of Biological Physics, vol. 129, no. 2-3, pp. 123-128, 2003. 